The primary disadvantage of the system is the 75-lb. (34-kg.) weight of a carboy containing 30 litres of dialysate. To protect employees from back injuries, we have insisted that the filled bottles be lifted each time by two men.

\section{ECONOMY OF Method}

The cost of producing the dialysis solutions is approximately one-fourth that of proprietary dialysis fluids. This includes the price of labour, but not that of laboratory determinations for quality control. The total price of the initial equipment such as mixingtanks, a pump, filter holders, and a dozen carboys is about $£ 625(\$ 1,500)$. Many hospital pharmacies carry these items as part of their standard equipment.

\section{RERERENCBS}

Boen, S. T. (1961). Medicine (Baltimore), 40, 243. Boen, S. T., Mion, C. M., Curtis, F. K., and intern. Organs, 10, 409 .

Cohen, S. L., and Percival, A. (1968). Brit. med.

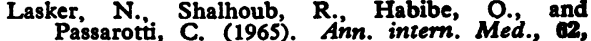
1147

Levin, S., and Winkelstein, J. A. (1967). Niw Engl. भ. Med., 277, 619. Palmer, R. A., Newell, J. B., Gray, E. J., and
Quinton, w. E. (1966). New Engl. f. Med., 274, 248 .

Ribot, S., Jacobs, M. G., Frankel, H. J., and Bernstein, A. (1966). Amer. F. med. Sci., 252,

Stevens, R. E:, Baskin. S., Greene, J. A., and Weller, J. M. (1964). f. Amer. med. Ass., 190, 1128 .

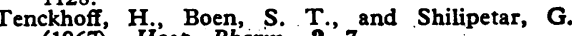
(1967). Hosp. Pharm., 2, 7. D. Y. (1967). F. Amer., med. Ass., $200,97$.

Weston, $\dot{R}$. E. and Roberts, M. (1965), Arch. intern. Med., 115, 659.

\title{
Mobile Bath for Severely Incapacitated or Completely Bedridden Patient
}

Dr. D. H. BlAKE, medical superintendent and specialist geriatrician, and $\mathrm{Mr}$. A. H. JoHnson, purchasing officer, of the Bendigo Home and Hospital for the Aged, Bendigo, Victoria, Australia, write: All those concerned with the care of the geriatric patient will be aware of the difficulty and sometimes complete impossibility of bathing the severely incapacitated or completely bedridden patient. The mobile bath has been designed to make possible the bathing of even the most helpless patient, and at the same time render the procedure one that can be relatively easily carried out by the nursing staff.

Hitherto, extreme difficulties have been encountered in hospitals in bathing these incapacitated patients, such bathing usually requiring manual lifting from bed to wheelchair or other carrying device, thence to a bath. Hydraulically actuated hoists are relatively expensive and are generally used in conjunction with other equipment, the patient thereby experiencing the feeling of insecurity brought about by the manner in which he must be moved. The project was therefore to provide a unit which is relatively simple and inexpensive, which is convenient to use, and which would involve the minimum of discomfort to the patient.

Description.-As can be seen from the photographs, the mobile bath is basically a wheeled trolley having a collapsible bath mounted on the top (Fig. 1).: The trolley is so constructed that it can be wheeled over an island-type fixed bath in the ward bathroom. This means that, if desired, the bath can be filled from a thermostatically controlled mixer tap at the head of the fixed bath and be. emptied into the fixed bath via the drain plug provided. The trolley has four braked

castors, and its top is made to standard bed height. The bath is manufactured from a heavy nylonized polyvinyl chloride material with waterproof seams. This fabric is held over a padded former and is raised by springloaded tubes held to the trolley frame. This former is locked in the up or down position by four synchronized shoot bolts.

bed wire from each end of the trolley. The patient can now be moved very easily from the bed to the trolley by a Masson roller, a draw sheet being used as the medium of transfer. When the patient is in the correct position on the trolley the lever at the side is released, allowing the bath to rise to its fixed position. The patient can then be

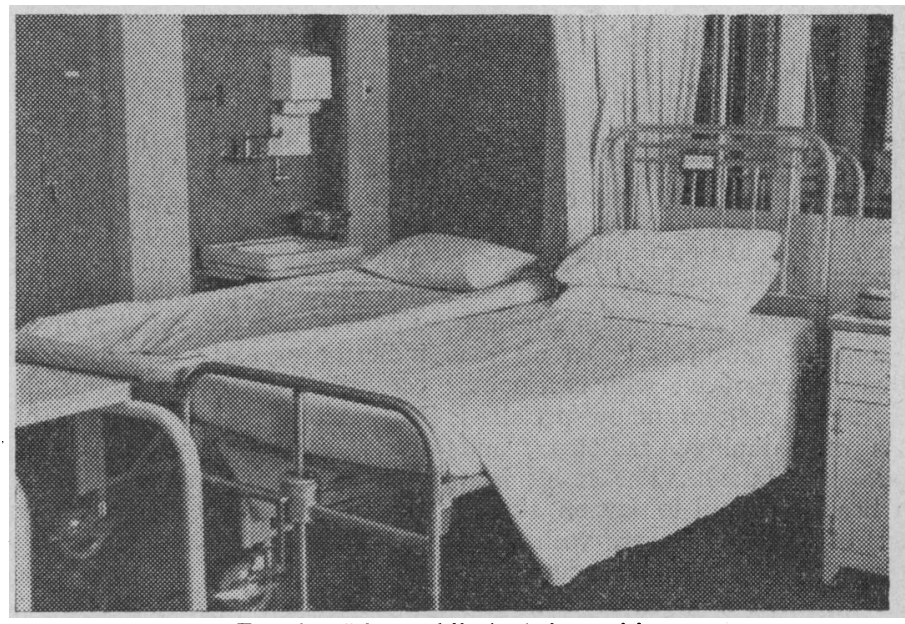

Fig. 2.-The mobile bath in position.
Method of Use.-This is quite simple. By releasing the lever at the side of the trolley the top former can be depressed to allow the sides of the bath to collapse. This gives an almost flat surface on top of the trolley, which is pushed up to the side of the patient's bed with the drain plug end at the patient's feet (Fig. 2). Safety chains are then connected to the under side of the

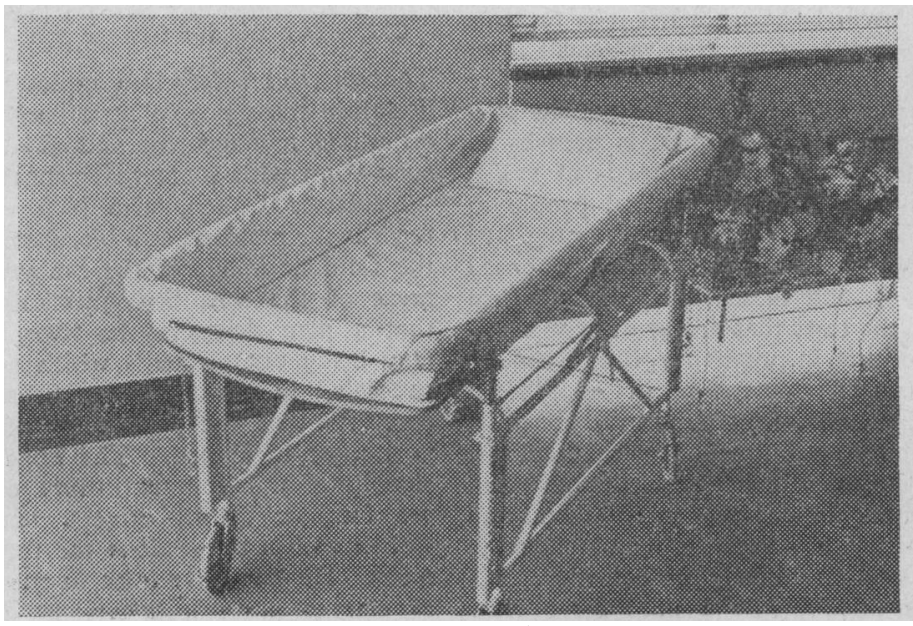

transported safely and by one person to the bathroom. Once the trolley is in position the patient can be undressed and bathed. We find that a temperature-controlled mixing-valve with an extended hose-shower head is very satisfactory. The plug can be left in or out as desired. Nursing staff do not have to bend, and can bath the patient from a standing position. After bathing, the whole procedure is reversed in transferring the patient back to bed.

As the patient is actually lying in the bath when it is filled, we would stress the necessity of using a thermostatically controlled mixingvalve. This is particularly necessary, as the type of patient involved may have lesions affecting the sensory system. The material used allows the inside of the bath to be quickly dried, so that once the patient has been towelled he can be dressed, while still in the bath, before transporting him back to the ward.

A prototype of the bath, for which patent is pending, has been subjected to trial in our wards and has proved to be a valuable additional nursing aid. Both patients and nursing staff have appreciated its assets. Nursing staff have easily become familiar with its use, and have employed it increasingly. and for a greater variety of patients. 